\title{
SUBMILLIMETER IMAGING POLARIMETRY OF THE NGC 7538 REGION
}

\author{
M. Momose, ${ }^{1,2}$ M. Tamura, ${ }^{3}$ O. Kameya, ${ }^{3,4}$ J. S. Greaves, ${ }^{5}$ A. Chrysostomou, ${ }^{5,6}$ J. H. Hough, ${ }^{6}$ AND J.-I. Morino ${ }^{7}$ \\ Received 2000 September 5 ; accepted 2001 March 20
}

\begin{abstract}
Imaging polarimetry of the $850 \mu \mathrm{m}$ continuum emission in the NGC 7538 region, obtained with the SCUBA Polarimeter, is presented. The polarization map is interpreted in terms of thermal radiation by magnetically aligned dust grains. Two prominent cores associated with IRS 1 and IRS 11, IRS 1(SMM), and IRS 11(SMM), are found in the surface brightness map. Although these cores look similar in surface brightness, their polarization shows striking differences. In IRS 11(SMM), the polarization vectors are extremely well-ordered, and the degrees of polarization are quite high with an average of $\sim 3.9 \%$. In IRS $1(\mathrm{SMM})$, on the other hand, the directions of polarization vectors are locally disturbed, and the degrees of polarization are much lower than those of IRS 11(SMM). These differences suggest that small scale fluctuations of the magnetic field are more prominent in IRS 1(SMM). This can be interpreted in terms of the difference in evolutionary stage of the cores. Inside IRS 1(SMM), which seems to be at a later evolutionary stage than IRS 11(SMM), substructures such as subclumps or a cluster of infrared sources have already formed. Small scale fluctuations in the magnetic field could have developed during the formation of these substructures. The distribution of magnetic field directions derived from our polarization map agrees well with those of molecular outflows associated with IRS 1(SMM) and IRS 11(SMM). Comparisons of energy densities between the magnetic field and the outflows show that the magnetic field probably plays an important role in guiding the directions of the outflows.

Subject headings: instrumentation: polarimeters — ISM: clouds — ISM: individual (NGC 7538) -

ISM: magnetic fields - stars: formation - submillimeter

On-line material: machine-readable table
\end{abstract}

\section{INTRODUCTION}

Outflows and jets are ubiquitous in young stellar objects (YSOs). Since the discovery of the first bipolar outflows in the 1980's (Snell et al. 1980), their study has become one of the central topics of star formation. It is suspected that outflows play a crucial role in removing angular momentum and magnetic fields and so are natural consequences of star formation processes (Shu, Adams, \& Lizano 1987). However, in spite of the major developments in revealing the dynamics of a large number of outflows by radio/ millimeter-wave observations, the details of driving and collimating mechanisms of bipolar outflows are still controversial even if the magnetohydrodynamical models are in consideration (see a review by Königl \& Ruden 1993). One of the important reasons why we have not been able to confidently judge these models with observations is the lack of information of the magnetic field structure near YSOs. The regions near YSOs are usually highly obscured by the embedding molecular cloud as well as the circumstellar matter such as envelopes or cloud cores. Therefore, conventional "interstellar polarimetry" at optical or near-infrared wavelengths cannot be employed to determine the field near

\footnotetext{
${ }^{1}$ Institute of Astrophysics and Planetary Sciences, Ibaraki University, Bunkyo 2-1-1, Mito, Ibaraki 310-8512, Japan.

2 momose@mito.ipc.ibaraki.ac.jp.

${ }^{3}$ National Astronomical Observatory of Japan, Osawa, Mitaka, Tokyo 181-8588, Japan.

${ }^{4}$ Mizusawa Astrogeodynamics Observatory, Mizusawa, Iwate 0230861, Japan.

${ }^{5}$ Joint Astronomy Centre, 660 North A'ohōkū Place, University Park, Hilo, HI 96720.

${ }^{6}$ Department of Physical Sciences, University of Hertfordshire, Hatfield, Hertfordshire AL10 9AB, UK.

${ }^{7}$ Nobeyama Radio Observatory, Nobeyama, Minamisaku, Nagano 384-1305, Japan.
}

YSOs. The most direct technique for mapping the magnetic fields near YSOs is to observe the polarized emission from dust at far-infrared or submillimeter wavelengths. Such techniques have become very efficient and powerful with use of large telescopes and array detectors that enable imaging polarimetry rather than point-by-point polarization mapping (e.g., Hildebrand 1996; Davis et al. 2000; Matthews \& Wilson 2000; Schleuning et al. 2000).

This paper presents polarization maps of the $850 \mu \mathrm{m}$ continuum emission in the NGC 7538 region with the SCUBA Polarimeter (SCUPOL). The NGC 7538 molecular cloud is an active site of high-mass star formation containing several pointlike infrared sources (Werner et al. 1979), and there are three prominent molecular outflows associated with these infrared sources (Kameya et al. 1989). Two remarkable submillimeter/infrared sources, IRS 1 and IRS 11 , are located in our mapped area. The inclination angle of the gaseous ring $0.13 \mathrm{pc}$ in radius around IRS 1 has been estimated to be $37^{\circ}$ from high-resolution imaging (Kawabe et al. 1992), hence the outflow associated with IRS 1 is likely $\sim 30^{\circ}$ from the line of sight. The inclination angle of the IRS 11 outflow is less certain, but its spatial distribution is similar to that of the IRS 1 outflow (Kameya et al. 1989, 1990a), suggesting it is also around $30^{\circ}$. Point-by-point polarization measurements of the submillimeter continuum emissions from IRS 1 and IRS 11 were made by Flett \& Murray (1991) and Minchin \& Murray (1994). Their measurements suggested that the magnetic fields near these infrared sources are parallel to the directions of their outflows. Our polarization maps obtained with SCUPOL have revealed the internal magnetic field structure of the cloud cores surrounding IRS 1 and IRS 11 with unprecedented details, allowing us to discuss the relationship between the magnetic field and the outflows associated with infrared sources in this region. 
TABLE 1

Flux Densities, Polarization Degrees, AND POSITION ANGLES

This table is available only on-line as a machine-readable table

The outline of this paper is as follows. Our observations and the procedure of data reduction are described in $\S 2$. In $\S 3$ we present our polarization/magnetic field maps and compare them with outflow distribution. The physical connection of the magnetic field structure with the evolution of cores and the outflows is discussed in $\S 4$.

\section{OBSERVATIONS AND DATA REDUCTION}

\subsection{Observations and Data}

The data were obtained with the James Clerk Maxwell Telescope (JCMT) atop Mauna Kea in Hawaii on 1998 July 18 (HST). The beam size was 14" (FWHM) at $850 \mu \mathrm{m}$. Polarimetry was conducted with a rotating half-wave plate and a fixed wiregrid analyzer in front of the SCUBA (Submillimeter Common-User Bolometer Array) window. Further details of the polarimeter, SCUPOL, are described by Greaves et al. (2000). Jiggle maps were obtained at waveplate positions 22.5 apart, repeated 8 times so that a sufficient signal-to-noise ratio was achieved. The atmospheric optical depth at $225 \mathrm{GHz}$, as measured by a tipping radiometer at CSO, was about 0.17 and stable during the observations. The air mass was $\sim 1.4$, and the seeing was 0.3 at $350 \mathrm{GHz}$. Chopping was $120^{\prime \prime} \mathrm{EW}$. Unfortunately, the bright and compact submillimeter source IRS 9, whose peak intensity is $\sim 25 \%$ of the IRS 1 peak, was located in the reference field, and some parts of the resultant maps of surface brightness and polarization vectors were corrupted. We shall describe its effects in detail when we present our results in $\S 3$.

\subsection{Reduction}

The ORAC-DR reduction program package was used for flat-fielding, extinction correction, sky-noise removal, badpixel removal, and rebinning. The sky removal was made from the median of the signal from the outermost bolometers that seemed emission-free in a short integration map. The polarization calibration including subtraction of instrumental polarization of $\sim 1 \%$ and debiasing, as well as plotting polarization vectors were also made using this package. The pixel size of the surface brightness map and the maps of the Stokes $Q$ and $U$ parameters were originally taken to be $3^{\prime \prime}$, but the polarization vectors were derived from data binned into $9^{\prime \prime}$ pixels. The resultant polarization data for positions at which the flux density is positive and the polarization degree is less than $10 \%$ are given in Table 1. Flux calibration was made using the brightness at the position of IRS 1, 19 Jy per 17".5 beam (Sandell 1994), corresponding to $493 \mathrm{mJy}$ per $3^{\prime \prime}$ pixel in the surface brightness map. Sky noise removal using the outermost bolometers causes a systematic error in flux measurement, but its amount is estimated to be only $\sim 15 \mathrm{mJy}$ per $3^{\prime \prime}$ pixel.

\section{RESULTS}

\section{1. $850 \mu \mathrm{m}$ Continuum Emission}

Figure 1 shows the surface brightness map of the $850 \mu \mathrm{m}$ continuum emission. Strong submillimeter continuum emissions associated with IRS 1 and IRS 11 are clearly seen.
Each core is resolved with the SCUBA beam and their shape is nonspherical, but being elongated with several protrusions. The northern core [hereafter denoted by IRS $1(\mathrm{SMM})]$, which contains IRS 1 , is associated with three protrusions to the east, to the west and to the southwest. These positionally coincide with the local peaks (CS2, CS4, and CS5, respectively) in a high resolution (6" beam) map of the CS $(J=2-1)$ line emission by Kawabe et al. (1992). The southern core [hereafter denoted by IRS 11(SMM)], which contains IRS 11 and has been much less studied, is associated with three protrusions to the east, to the southwest and to the northwest.

Previous observations with submillimeter dust continuum as well as with the $\mathrm{HCN}$ and $\mathrm{HCO}^{+}$lines (Minchin \& Murray 1994; Cao et al. 1993; Kameya et al. 1990a and unpublished data) revealed faint emissions connecting two cores associated with IRS 1 and IRS 11. Although the existence of such emissions is also suggested in our surface brightness map, the position corresponding to IRS 9 in the reference beam is located just between the two cores (see Fig. 1), corrupting part of the emissions connecting these two cores. Previous submillimeter continuum observations with the JCMT have revealed that the emissions associated with IRS 9 is so compact that the corrupted regions are expected to be approximately one beam (Greaves et al., unpublished data). Therefore, the spatial distributions of the core emissions in Figure 1 are hardly distorted, while detailed structure of the emissions between the cores cannot be discussed in this paper.

The total flux density of the continuum emission is 111 Jy, and the flux densities for IRS 1(SMM) and IRS $11(\mathrm{SMM})$ measured in the regions where the brightness is greater than $58.5 \mathrm{mJy}$ per $3^{\prime \prime}$ pixel are 65 and $29 \mathrm{Jy}$, respectively. Assuming that the continuum emission is optically thin and the dust temperature $T_{d}$ is uniform, one can estimate the cloud mass by

$$
M=\frac{F_{\lambda} d^{2}}{\kappa_{\lambda} B_{\lambda}\left(T_{d}\right)},
$$

where $\kappa_{\lambda}$ is the mass absorption coefficient by dust grains, $B_{\lambda}(T)$ is the Planck function, $F_{\lambda}$ is the flux density of the continuum emission, and $d$ is the distance to these cores. With $T_{d}=25 \mathrm{~K}$ (see Dickel, Dickel, \& Wilson 1981), $d=2.7$ kpc (IAU Trans., 12B, 351 [1964]) and $\kappa_{850 \mu \mathrm{m}}=8.65$ $\times 10^{-3} \mathrm{~cm}^{2} \mathrm{~g}^{-1}$, which is obtained by the formula of Hildebrand (1983) with $\beta=2$, the total mass is derived to be $6.7 \times 10^{3} M_{\odot}$, and for IRS $1(\mathrm{SMM})$ and IRS $11(\mathrm{SMM})$ to be $3.9 \times 10^{3} M_{\odot}$ and $1.8 \times 10^{3} M_{\odot}$, respectively. The total mass described above is in fairly good agreement with that derived from the CS and $\mathrm{C}^{34} \mathrm{~S}(J=1-0)$ mapping observations by Kameya et al. (1986), though our measurement missed part of the faint emission components connecting the two cores.

\section{2. $850 \mu \mathrm{m}$ Continuum Polarization and Magnetic Field}

Figures $2 a$ shows the map of the $850 \mu \mathrm{m}$ polarization vectors superposed on the surface brightness map. Since the $850 \mu \mathrm{m}$ continuum emission from this region is thermal radiation from dust, the polarization is due to the emission from magnetically aligned dust grains (Davis \& Greenstein 1951). In Figure $2 b$, the polarization vectors are rotated by $90^{\circ}$ so that it directly shows the magnetic field direction projected on the sky. The polarization vectors in the main 


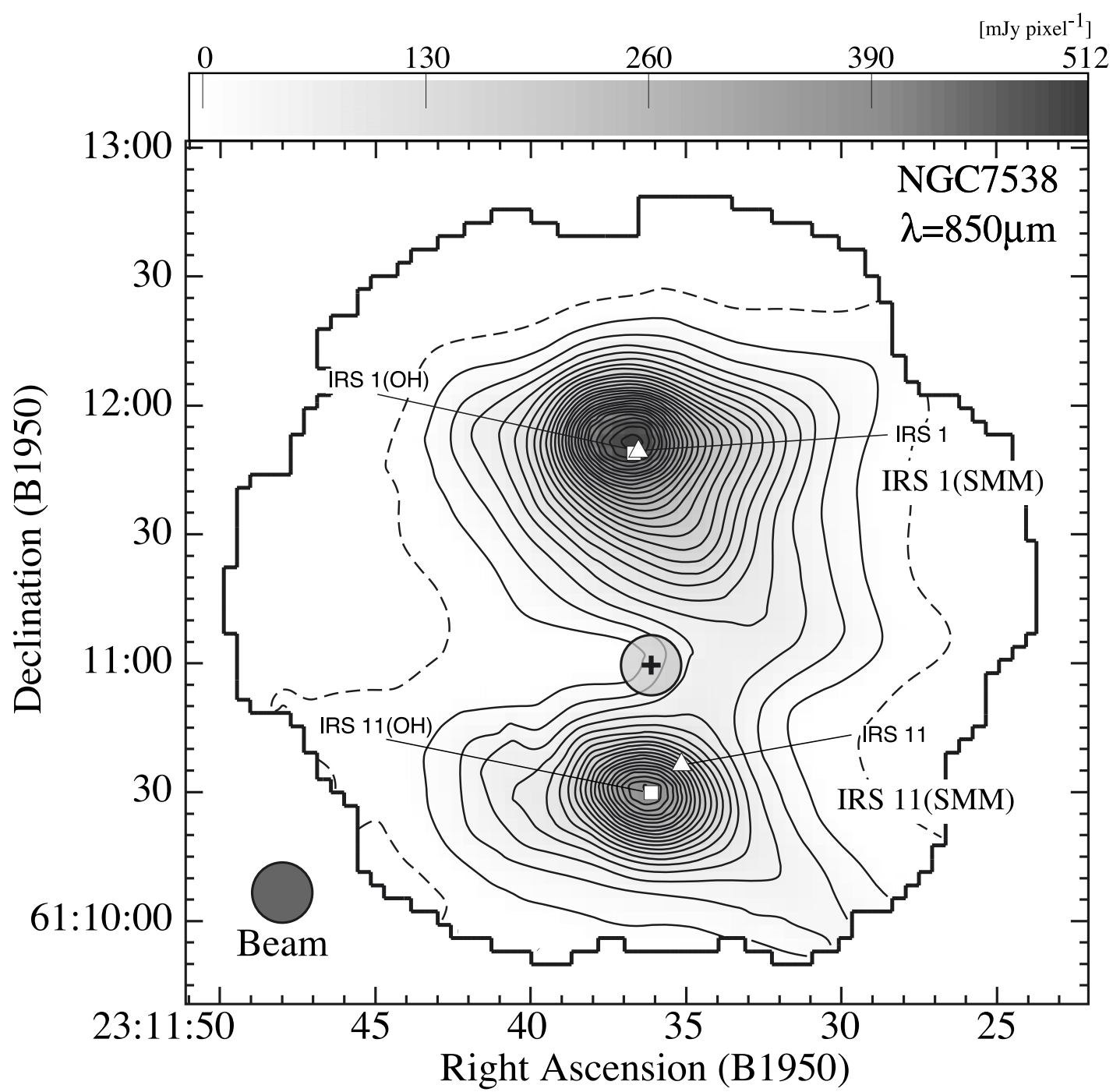

FIG. 1.-The surface brightness map of the $850 \mu \mathrm{m}$ continuum emission in the NGC 7538 region. The pixel size is $3^{\prime \prime} \times 3^{\prime \prime}$ and the contour spacing is 19.5 mJy pixel ${ }^{-1}$, starting at $9.75 \mathrm{mJy}_{\text {pixel }}{ }^{-1}$. The dashed lines indicate the level of $-9.75 \mathrm{mJy} \mathrm{pixel}^{-1}$. The heavy line indicates the field of view. Triangles and squares show the positions of infrared sources (Werner et al. 1979) and OH masers (Wynn-Williams, Werner, \& Wilson 1974b), respectively. The gray circle with a black cross in the map indicates the one-beam (FWHM) area around IRS 9 in the reference beam.

bodies of the cores systematically change their amplitudes and directions, indicating that the internal field structure of these two cores is successfully revealed. However, the contamination from IRS 9 in the reference field does not allow us to discuss the field structure in the regions between the cores. In addition, polarized emission components from side lobes of the beam, which cause error in polarization measurement for fainter regions of extended sources, cannot be removed. Polarized components from side lobes can be as strong as $0.1 \%$ of the peak intensity within or near the field of view (Greaves et al. 2000, SCUBA Polarimeter Commissioning Results in Semester 99B and 00A). We shall therefore use polarization data where the flux density is higher than $10 \%$ of the IRS 1 (SMM) peak when we give quantitative description of the field structure.

The surface brightness map of the continuum emission gives an impression that the region is comprised of two similar cores, but the magnetic field map reveals clear contrast between IRS 1(SMM) and IRS 11(SMM). Therefore, we shall describe the details of the magnetic field structure of each region and its relationship with the outflow from the central sources.

\subsubsection{IRS 11(SMM)}

As seen in Figure $2 b$, the magnetic field structure over IRS $11(\mathrm{SMM})$ is extremely well ordered. Figure $3 a$ shows the histogram of the magnetic field direction in IRS $11(\mathrm{SMM})$ where the flux density is higher than $10 \%$ of the IRS 1 (SMM) peak (24 points). Prominent is the $170^{\circ}$ component, which dominates the region to the south of IRS 11. As one moves to the north, its direction smoothly changes over IRS 11 and is pointed to the west. A simple histogram of the field direction does not reveal the coherent and gradual change of the field direction. Therefore, we have calculated an average by rows and subtracted the average from each point of the rows. The resultant histogram of the local "deviation" of the field is shown in Figure 4a. The histogram is symmetric around the peak with small variance, showing the coherence or uniformity of the magnetic field in IRS 11(SMM).

The smoothly bent but locally uniform magnetic field is in extremely good agreement with the morphology of the CO $J=3-2$ bipolar outflow (Kameya et al. 1990a), as shown in Figure $2 b$. The $\mathrm{CO}$ outflow appears roughly 


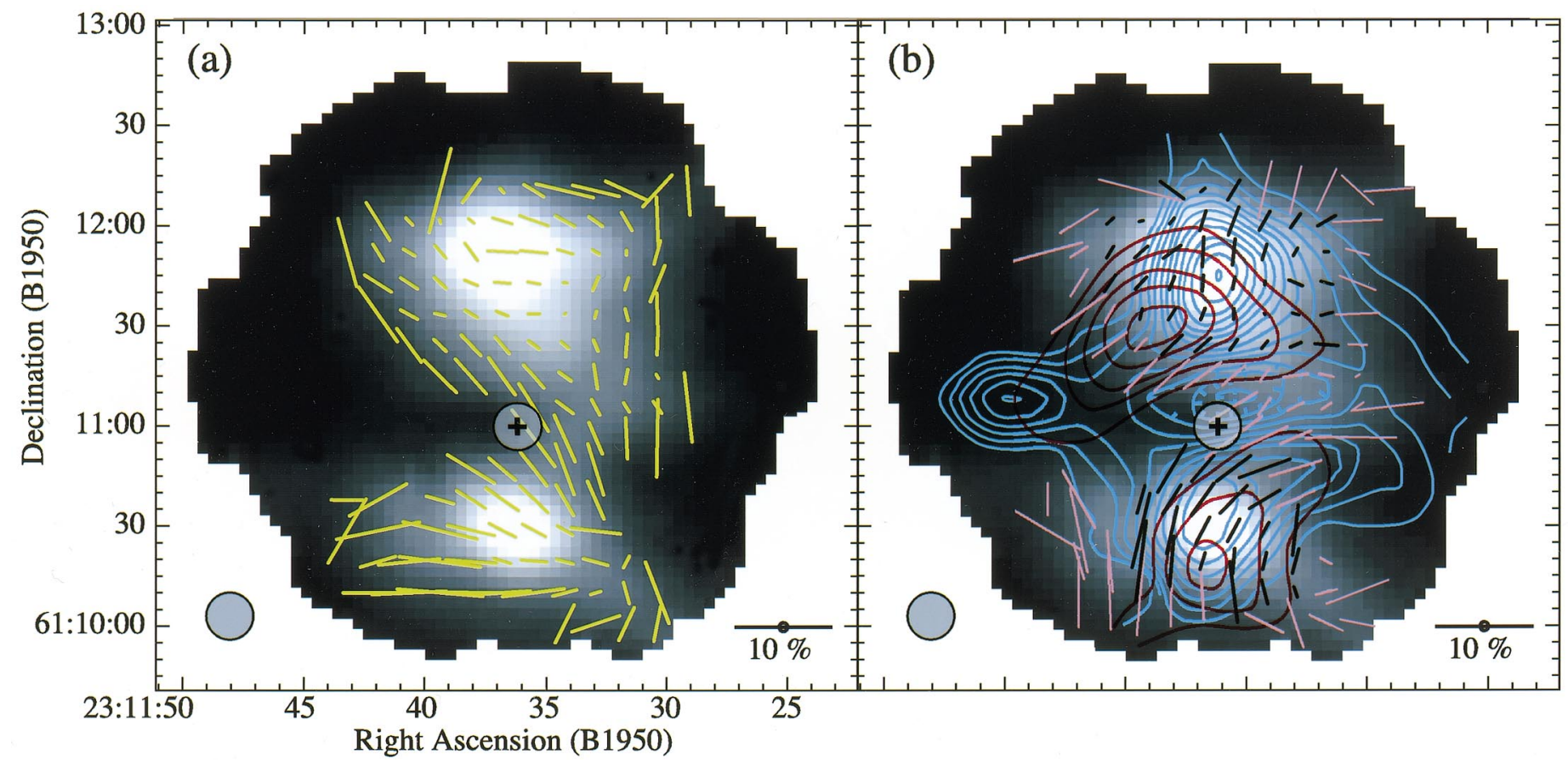

Fig. 2.-(a) Polarization $E$ vectors of the $850 \mu \mathrm{m}$ continuum emissions (thick lines) superposed on the surface brightness map shown in Figure 1 (gray scale). In total, 122 data points are plotted. The length of each line is proportional to the polarization degree. (b) Magnetic field directions derived from the polarization vectors in (a) (black and violet lines) superposed on the distribution of $\mathrm{CO}(J=3-2)$ high-velocity outflows obtained by Kameya et al. (1990a) (contours) and the $850 \mu \mathrm{m}$ surface brightness map (gray scale). The black lines indicate field directions inside the regions where the flux density is higher than $10 \%$ of the IRS 1 (SMM) peak while the violet lines indicate those outside the regions. Only the data shown by black lines are used when the quantitative description of the field structure is made. The blue and red contours show the distribution of the $\operatorname{CO}(J=3-2)$ emissions integrated over $v_{\text {lsr }}=-74$ to -64 $\mathrm{km} \mathrm{s}^{-1}$ and $v_{1 \mathrm{sr}}=-54$ to $-44 \mathrm{~km} \mathrm{~s}^{-1}$, respectively. The gray circle with a black cross in each map indicates the one-beam (FWHM) area around IRS 9 in the reference beam.

bipolar to the south of IRS 11, consistent with the northsouth field direction in this area. In particular, the outflow to the north, or the blueshifted component, gradually bends to the west, showing good correlation with the smoothly bent magnetic field. These facts suggest either that the magnetic field controls the directions of the outflows or that the magnetic field structure is influenced by the outflow dynamics. We shall discuss further on this point in $\S 4.2$.

Figure $5 a$ shows the histogram of the degrees of polarization. The degrees of polarization over IRS 11(SMM) are very high, ranging from 1 to $9 \%$ with an average of $3.9 \%$. This polarization level is higher than the typical percentage of "on-peak" submillimeter polarization of star-forming regions observed with the same beam of the JCMT $\left(\sim 14^{\prime \prime}\right.$, Tamura et al. 1999; Vallée \& Bastien 2000) and is much

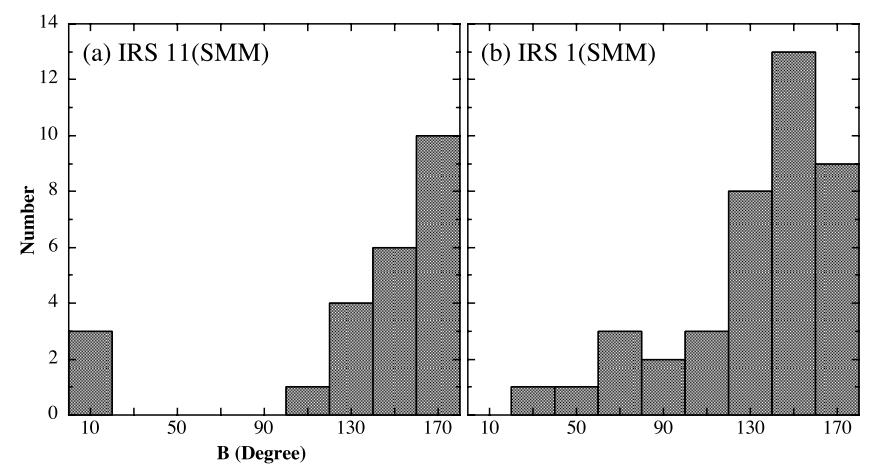

FIG. 3.- (a) Histogram of the magnetic field directions (i.e., the position angles of $850 \mu \mathrm{m}$ polarization plus $90^{\circ}$ ) in IRS $11(\mathrm{SMM})$. (b) Same as $(a)$ but in IRS 1(SMM).

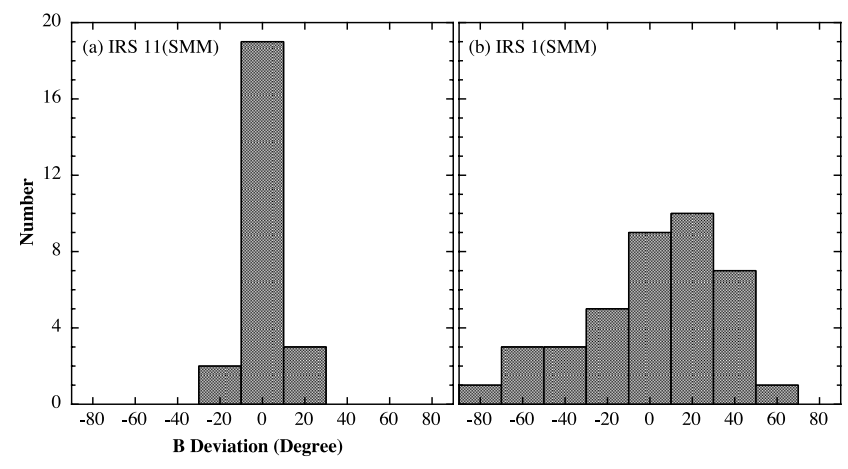

FIG. 4. - (a) Histogram of the deviations of the magnetic field direction from an average of a local magnetic field in IRS 11(SMM). (b) Same as (a) but in IRS 1(SMM).

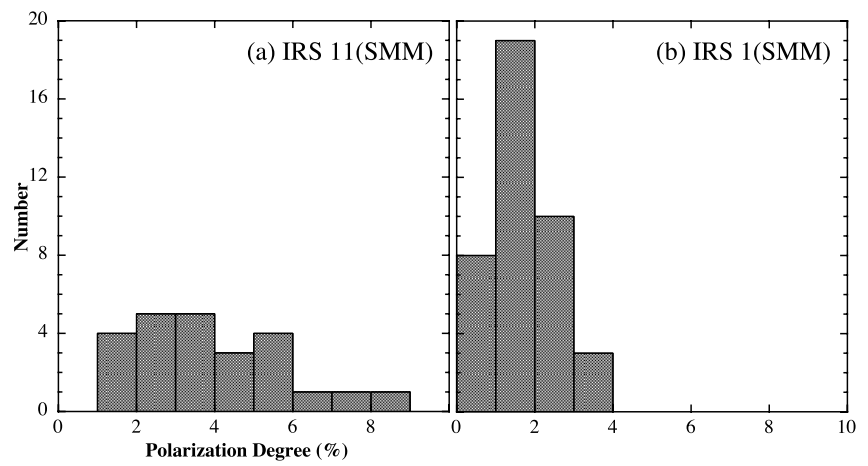

FIG. 5.-(a) Histogram of the polarization degrees in IRS 11(SMM). (b) Same as $(a)$ but in IRS 1(SMM). 
higher than that of IRS 1(SMM) (see $\S 3.2 .2)$. The degree and position angle of the polarized emission at the peak of IRS 11(SMM) obtained from our data are $(3.0 \pm 0.1) \%$ and $\sim 62^{\circ}$. These values are in good agreement with the $800 \mu \mathrm{m}$ polarization obtained by Flett \& Murray (1991) $\left(3.8 \% \pm 1.0 \%\right.$ and $\left.52^{\circ} \pm 8^{\circ}\right)$ or Minchin \& Murray (1994) $\left(2.5 \% \pm 0.2 \%\right.$ and $\left.58^{\circ} \pm 2^{\circ}\right)$ with the JCMT.

\subsubsection{IRS 1(SMM)}

The dominant magnetic field component over IRS $1(\mathrm{SMM})$ is the field running from northwest to southeast. The median of the distribution of the magnetic field direction in IRS 1(SMM) where the flux density is higher than $10 \%$ of the IRS 1 (SMM) peak (Fig. $3 b ; 40$ points) is $\sim 150^{\circ}$. The degrees of polarization in this region range from $0 \%$ to $4 \%$ with an average of $1.6 \%$ (Fig. $5 b$ ). This level of polarization has typically been observed in a number of starforming regions (e.g., Vallée \& Bastien 2000).

It is noteworthy that the dominant magnetic field is consistent with the direction of the large-scale $(\sim 0.3 \mathrm{pc}) \mathrm{CO}$ bipolar outflow (Fig. 2b, see also Fischer et al. 1985; Scoville et al. 1986; Kameya et al. 1989). The blueshifted component is to the northwest of IRS 1 and the redshifted component is to the southeast, both in the position angle of $\sim 135^{\circ}$. In contrast, the radio continuum emission originating from jets shows a gradual change of its direction: from $\sim 180^{\circ}$ on 0.005 pc scale to $\sim 165^{\circ}$ on 0.03 pc scale (Campbell 1984).

In addition to the dominant magnetic field, there are several fine structures seen in Figures $2 a$ and $2 b$. Most interesting is the presence of two small polarization regions on the opposite sides of IRS 1: $25^{\prime \prime}$ southwest of IRS 1 where the degree of polarization is as small as $0.2 \%$ and northeast of IRS 1 where the degrees of polarization are $\sim 0.5 \%$. This reminds us of the "polarization disks" that are often observed in the near-IR polarization maps of star-forming regions and are indicative of the disk plane and therefore perpendicular to the outflow direction (e.g., Tamura et al. 1991; Lucas \& Roche 1998). The direction of the "submillimeter polarization disk" in IRS 1(SMM) roughly coincides with the major axis of the disklike structure observed with molecular emissions (e.g., Scoville et al. 1986; Kawabe et al. 1992), although the origin of such polarization pattern at submillimeter wavelengths is not clear (for the case of a filamentary cloud, see Fiege \& Pudritz 2000).

Another intriguing feature of the magnetic field is the relative nonuniformity of the field over IRS 1(SMM), when compared with IRS 11(SMM). Although the total change of the magnetic field across IRS 11(SMM) is large (as large as $\sim 60^{\circ}$ ), the field direction change is gradual. In contrast, the overall field change in IRS 1(SMM) is not so large, but the field is locally more chaotic. This behavior is shown in the histogram of the local "deviation" of the field constructed in the same manner as that for IRS 11 (Fig. 4b). The histogram is asymmetric around the peak with larger variance.

The degree and position angle of the polarized emission at IRS 1 obtained from our data are $(3.0 \pm 0.2) \%$ and $\sim 87^{\circ}$. Flett $\&$ Murray (1991) observed $800 \mu \mathrm{m}$ polarization vector at the position $\sim 9^{\prime \prime}$ north of IRS 1 . Their polarization vector, $0.7 \% \pm 0.3 \%$ and $52^{\circ} \pm 15^{\circ}$, is not consistent with our data $\left(2.1 \% \pm 0.3 \%\right.$ and $72^{\circ} \pm 3^{\circ}$, see no. 108 in Table 1 ), but it agrees with our data for the position $9^{\prime \prime}$ east of their observed position $\left(0.8 \% \pm 0.3 \%\right.$ and $52^{\circ} \pm 10^{\circ}$, see no. 107 in Table 1). The possible pointing error in their measurements as well as their larger beam size (19" [FWHM], see Minchin \& Murray 1994) might cause the above inconsistency.

\section{DISCUSSION}

\subsection{Relationship between Core Evolution and Magnetic Field Structure}

Previous observations of the NGC 7538 region revealed that IRS 1(SMM) and IRS 11(SMM) commonly show features that can be regarded as the signs of ongoing star formation: huge far-infrared luminosities indicating the existence of embedded energy sources (Werner et al. 1979), bipolar outflows probably powered by those embedded young stellar objects (Kameya et al. 1989), and $\mathrm{H}_{2} \mathrm{O}$ maser spots closely associated with ultracompact $\mathrm{H}$ II regions (Kameya et al. 1990b). Infrared images taken at wavelengths shorter than $20 \mu \mathrm{m}$, however, manifest remarkable differences between these two cores. The point sources in IRS 1(SMM), as well as their surrounding nebulosity, can easily be identified at $2.2 \mu \mathrm{m}$, but only a faint extended emission is found in IRS 11(SMM) at the same wavelength (e.g., Davis et al. 1998). Even at 10-20 $\mu \mathrm{m}$ there is no detectable source in IRS 11(SMM) (Werner et al. 1979). These facts suggest that young stellar objects in IRS 11(SMM) are more deeply embedded compared with those in IRS $1(\mathrm{SMM})$ and that IRS 11(SMM) is in an earlier evolutionary stage of star formation. It is therefore expected that the comparison of polarization maps between these two cores will provide information about the change of internal magnetic-field structure during the course of core evolution.

The path of core evolution depends on whether its initial state is magnetically subcritical or supercritical. Although it is difficult to precisely measure the mass-to-flux ratio in cores with observations, the most massive cores seem to be in magnetically supercritical state, in which the magnetic force is not strong enough to support the core (Bertoldi \& McKee 1992; see also Crutcher 1999). Scott \& Black (1980) made detailed numerical calculations of the collapse of a magnetically supercritical core and found that the collapse occurs preferentially along the field direction to form a disklike subregion with higher density. The disklike subregion is likely to fragment further into smaller subclumps because this region has the same ratio of surface-density to magnetic-flux as the entire cloud and is still in a magnetically supercritical state (Scott \& Black 1980; see also Mestel 1985; Shu et al. 1987). Since the interstellar medium couples well with magnetic fields (unless the hydrogen number density is greater than $10^{11} \mathrm{~cm}^{-3}$ [Nakano \& Umebayashi 1986]), the magnetic field structure inside a core must strongly be affected by dynamical processes in the core described above. It is expected that small scale fluctuations of the magnetic field grow as substructures inside the core form.

Our polarization map shows striking differences between IRS 1(SMM) and IRS 11(SMM). The directions of polarization vectors in IRS 1(SMM) are locally disturbed while those in IRS 11(SMM) are well ordered, suggesting that small scale fluctuations of magnetic field are more prominent in IRS 1(SMM). The nonuniformity of polarization degree in IRS 1(SMM), which is not found in IRS 11(SMM), can also be explained by small scale fluctuations of the magnetic field; the observed polarization degree gets smaller if the field direction significantly changes within the beam size $\left(\sim 15^{\prime \prime}=0.2 \mathrm{pc}\right)$. Such differences in magnetic 
field structure can be interpreted in terms of the difference in evolutionary stage between these cores. Previous observations have shown that the formation of substructures inside IRS 1(SMM) is highly progressed: there is disklike structure with a radius of around $0.3 \mathrm{pc}$ (Scoville et al. 1986), and inside this structure several self-gravitating subclumps with a few tens of solar masses and $0.13 \mathrm{pc}\left(\sim 10^{\prime \prime}\right)$ in size have been found by aperture synthesis observations (Kawabe et al. 1992). Moreover, a cluster of infrared sources has already formed within the central regions of IRS 1(SMM) (Wynn-Williams, Becklin, \& Neugebauer 1974a). The formation of these substructures in IRS 1(SMM) would cause the development of small scale fluctuations in the magnetic field. Small scale variations of magnetic field direction have also been found in the Orion $\mathrm{BN} / \mathrm{KL}$ region, in which disklike structures and subclumps have also already formed (Burton et al. 1991; Chrysostomou et al. 1994). On the other hand, IRS 11(SMM) is expected to be in an earlier evolutionary stage compared with IRS 1(SMM). It is therefore likely that fragmentation in IRS 11(SMM) has not yet highly developed and that the magnetic field structure remains well ordered. Recent submillimeter observations of prestellar cores in the OMC-3 region, which is in an earlier stage of evolution compared with the Orion $\mathrm{BN} / \mathrm{KL}$ region (Chini et al. 1997), have revealed wellordered and high-degree of polarization vectors similar to those in the IRS 11(SMM) of NGC 7538 (Matthews \& Wilson 2000). We should keep in mind, however, that there are no observations which show the lack of prominent substructure inside IRS 11(SMM). Higher resolution observations of molecular gas toward IRS 11(SMM) are required in order to clearly reveal the relationship between the physical evolution of cores and magnetic field structure.

\subsection{Comparisons between the Polarization Map and Molecular Outflows}

The magnetic field directions derived from our polarization map are consistent with those of molecular outflows associated with IRS 1(SMM) and IRS 11(SMM) (see Fig. $2 b$ ). There are two possible explanations for this result: (i) the magnetic field strength in these cores is so strong that it controls the directions of the outflows, or (ii) the magnetic field strength is so weak that the field structure is influenced by the outflow dynamics. Comparisons of the energy density between the magnetic field and outflows are useful to judge which case is more plausible. If the magnetic field in each core has a greater energy density than the outflow, the former case is more plausible. Otherwise, the latter case is more plausible. Although this consideration might be too simplified, a more elaborate model for a low-mass outflow by Hurka, Schmid-Burgk, \& Hardee (1999), who made numerical calculations of the deflection of high-velocity jets by ambient magnetic field, gave a similar criterion for the maximum velocity of a jet that can be deflected by the ambient magnetic field.

The critical magnetic field strength at which the field has the same energy density as the outflows, $B_{\text {flow }}$, can be expressed by

$$
B_{\text {flow }}=\left(\frac{8 \pi E_{\text {flow }}}{V_{\text {flow }}}\right)^{1 / 2},
$$

where $E_{\text {flow }}$ and $V_{\text {flow }}$ are the total kinetic energy and volume of the outflow, which can be estimated from outflow observations (e.g., Kameya et al. 1989). Values of $B_{\text {flow }}$ for each core, as well as the physical parameters of the outflows, are summarized in Table 2. The magnetic field strengths in IRS 1(SMM) and IRS 11(SMM), on the other hand, can roughly be estimated from their column densities, though it cannot be derived directly from our observations. Using the data set compiled by Crutcher (1999), who examined the relationships between the magnetic field strengths in molecular clouds and their physical parameters, one can find the following relationship between the field strengths and column densities with its correlation coefficient of 0.85 :

$$
B=\frac{\pi}{2} B_{\text {los }}=229\left[\frac{N\left(\mathrm{H}_{2}\right)}{10^{23} \mathrm{~cm}^{-2}}\right]^{0.95} \mu \mathrm{G},
$$

where $B_{\text {los }}$ is magnetic field strength obtained by Zeeman measurements and $N\left(\mathrm{H}_{2}\right)$ is column density of a cloud. Using this equation the magnetic field strengths in IRS $1(\mathrm{SMM})$ and IRS $11(\mathrm{SMM})\left[N\left(\mathrm{H}_{2}\right) \approx 3.5 \times 10^{23} \mathrm{~cm}^{-2}\right.$, see fourth row in Table 2] are estimated to be $\sim 750 \mu \mathrm{G}$, which is greater than $B_{\text {flow }}$ (see eighth row in Table 2). It is there-

TABLE 2

Physical Parameters and Critical Magnetic Strengths for IRS 1(SMM) and

\begin{tabular}{|c|c|c|}
\hline Parameter & IRS 1(SMM) & IRS 11(SMM) \\
\hline (1) Spatial extent $\left(\mathrm{pc}^{2}\right)^{\mathrm{a}}$. & 0.657 & 0.358 \\
\hline (2) Mass $\left(M_{\odot}\right)^{\mathrm{a}} \ldots \ldots \ldots \ldots$ & $3.9 \times 10^{3}$ & $1.8 \times 10^{3}$ \\
\hline (3) Mean column density $\left(\mathrm{g} \mathrm{cm}^{-2}\right)$ & 1.24 & 1.05 \\
\hline (4) Mean column density $\left[N\left(\mathrm{H}_{2}\right]\right.$ in $\left.\mathrm{cm}^{-2}\right) \ldots \ldots$ & $3.7 \times 10^{23}$ & $3.2 \times 10^{23}$ \\
\hline 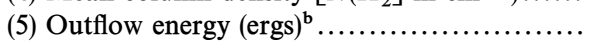 & $6 \times 10^{46}$ & $4 \times 10^{46}$ \\
\hline (6) Spatial extent of outflow $\left(\mathrm{pc}^{2}\right)^{\mathrm{b}} \ldots \ldots \ldots$ & 0.90 & 0.64 \\
\hline (7) Expected volume of outflow $\left(\mathrm{pc}^{3}\right)^{\mathrm{c}}$. & 0.85 & 0.51 \\
\hline 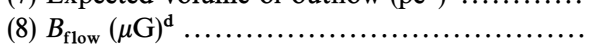 & 250 & 260 \\
\hline (9) $B_{\text {grav }}(\mu \mathrm{G})^{\mathrm{e}}$ & $2.0 \times 10^{3}$ & $1.7 \times 10^{3}$ \\
\hline
\end{tabular}
IRS 11(SMM)

${ }^{a}$ Derived from our observations. see $\S 3.1$.

${ }^{\text {b }}$ From Kameya et al. 1989.

c Estimated by (spatial extent) ${ }^{1.5}$.

d The critical field strength at which the field has the same energy density as the outflows, derived from eq. (2).

e The critical field strength of the cloud at which the magnetic force is comparable to the gravitational force, derived from $B_{\text {grav }}=2 \pi \sqrt{G} \Sigma$, where $G$ is the gravitational constant and $\Sigma$ is the column density of a cloud; see Nakano \& Nakamura 1978. 
fore plausible that the magnetic field guides the outflows on a larger scale. The field strength estimated from equation (3) is smaller than the critical field strength of each core at which the magnetic force is comparable to the gravitational force (see ninth row in Table 2). This is consistent with the fact that young stars have already formed in these cores, though the mechanical support from turbulent pressure is not taken into account when the critical field strength in the ninth row is estimated.

In addition to the large-scale outflows, the magnetic field direction in IRS 1(SMM) seems to be correlated with a jet on a smaller scale: although the dominant magnetic field in IRS 1(SMM) is running from northwest to southeast, the field near the emission peak is almost in the north-south direction (see Fig. $2 b$ ) which is consistent with the direction of the 0.03 pc scale jet ejected from IRS 1 (Campbell 1984). This fact could suggest that the magnetic field plays an important role in collimating small-scale jets, as has been discussed in many theoretical studies (e.g., Königl \& Ruden 1993). However, the angular resolution of our map is not sufficient to discuss this topic in detail. Polarimetry with higher angular resolution is desired to obtain more convincing evidence for the small scale collimation.

One may suspect that the small scale fluctuations of the polarization vectors in IRS 1(SMM) could be related to the outflows ejected from young stellar objects inside the core. However, this seems unlikely because the physical parameters of the outflow in IRS 1(SMM) are not so different from those of the outflow in IRS 11(SMM) (see Table 2) where the small scale fluctuations of magnetic field are not found.

\subsection{Relationship between Cores and Galactic Magnetic Field}

The direction of the dominant magnetic field component in IRS $1(\mathrm{SMM})$ and IRS $11(\mathrm{SMM})$ (position angle $\sim 160^{\circ}$; see Figs. $3 a$ and $3 b$ ) does not align with the interstellar polarization which can be regarded as the direction of galactic magnetic field (position angle $\sim 60^{\circ}$, see Dyck \& Lonsdale 1979). This fact may suggest that nonmagnetic forces such as self-gravity of the interstellar medium or compression by the nearby H II region (Kameya \& Takakubo 1988) regulate the formation processes of these cores and that the magnetic field inside the cores has experienced complete restructuring during their formation.

\section{CONCLUSIONS}

We have made imaging polarimetry of the $850 \mu \mathrm{m}$ continuum emission in the NGC 7538 region with the SCUBA Polarimeter mounted on the JCMT. The polarization maps are interpreted in terms of thermal radiation by magnetically aligned dust grains. Our conclusions are summarized as follows:
1. Two prominent cores associated with IRS 1 and IRS 11 [IRS 1(SMM) and IRS 11(SMM)] are found in the surface brightness map of the continuum emission. The total cloud mass derived from our surface brightness map is $6.7 \times 10^{3} M_{\odot}$.

2. The polarization map shows striking difference between IRS 1(SMM) and IRS 11(SMM). In IRS 11(SMM), the polarization vectors are extremely well ordered, and the degrees of polarization are very high, ranging from $1 \%$ to $9 \%$ with an average of $3.9 \%$. In IRS 1 (SMM), on the other hand, the directions of polarization vectors are locally disturbed, and the degrees of polarization range from $0 \%$ to $4 \%$ with an average of $1.6 \%$, which is much lower than that of IRS 11(SMM). These differences suggest that small scale fluctuations of magnetic field are more prominent in IRS 1(SMM).

3. Such differences in magnetic field structure can be interpreted in terms of a difference in evolutionary stage between these cores. Inside IRS 1(SMM) substructures such as subclumps or a cluster of infrared sources have already formed. Small scale fluctuations in the magnetic field can develop during the formation of these substructures. On the other hand, IRS 11(SMM) has no detectable source at wavelengths shorter than $20 \mu \mathrm{m}$ and seems to be in an earlier evolutionary stage compared with IRS 1 (SMM). It is therefore likely that fragmentation in IRS 11(SMM) has not yet sufficiently progressed and that the magnetic field structure remains well ordered.

4. The magnetic field directions derived from our polarization map agree well with those of molecular outflows associated with IRS 1(SMM) and IRS 11(SMM). Although this fact suggests either that the magnetic field controls the directions of the outflows or that the magnetic field structure is influenced by the outflow dynamics, comparisons of energy densities between the magnetic field and the outflows show that the former case is more plausible.

The JCMT is operated by the Joint Astronomy Centre, on behalf of the UK Particle Physics and Astronomy Research Council, the Netherlands Organization for Pure Research, and the National Research Council of Canada. We would like to thank N. Kaifu, K. Kodaira, S. Sato, and A. Boksenberg for promoting the UK-Japan collaboration. This work was supported in part by the grant-in-aid for Scientific Research of Ministry of Education, Science, Sports and Culture, and by the Japan Society of the Promotion of Science.

\section{REFERENCES}

Bertoldi, F., \& McKee, C. F. 1992, ApJ, 395, 140

Burton, M. G., Minchin, N. R., Hough, J. H., Aspin, C., Axon, D. J., \& Bailey, J. A. 1991, ApJ, 375, 611

Campbell, B. 1984, ApJ, 282, L27

Cao, Y. X., Zeng, Q., Deguchi, S., Kameya, O., \& Kaifu, N. 1993, AJ, 105, 1027

Chini, R., Reipurth, B., Ward-Thompson, D., Bally, J., Nyman, L.-A., Sievers, A., \& Billawala, Y. 1997, ApJ, 474, L135

Chrysostomou, A., Hough, J. H., Burton, M. G., \& Tamura, M. 1994, MNRAS, 268, 325

Crutcher, R. M. 1999, ApJ, 520, 706

Davis, C. J., Chrysostomou, A., Matthews, H. E., Jenness, T., \& Ray, T. P. 2000, ApJ, 530, L115

Davis, C. J., Moriarty-Schieven, G., Eislöffel, J., Hoare, M. G., \& Ray, T. P. 1998, AJ, 115, 1118

Davis, L., \& Greenstein, J. L. 1951, ApJ, 114, 206

Dickel, H. R., Dickel, J. R., \& Wilson, W. J. 1981, ApJ, 250, L43

Dyck, H. M., \& Lonsdale, C. J. 1979, AJ, 84, 1333

Fiege, J. D., \& Pudritz, R. E. 2000, ApJ, 544, 830

Fischer, J., Sanders, D. B., Simon, M., \& Solomon, P. M. 1985, ApJ, 293, 508

Flett, A. M., \& Murray, A. G. 1991, MNRAS, 249, 4P

Greaves, J. S., Jenness, T., Chrysostomou, A. C., Holland, W. S., \& Berry, D. S. 2000, in Imaging at Radio through Submillimeter Wavelengths, ed. J. Mangum \& S. Radford (San Francisco: ASP), 151

Hildebrand, R. H. 1983, QJRAS, 24, 267 
Hildebrand, R. H. 1996, in ASP Conf. Ser. 97, Polarimetry of the Interstellar Medium, ed. W. G. Roberge \& D. C. B. Whittet (San Francisco: ASP), 254

Hurka, J. D., Schmid-Burgk, J., \& Hardee, P. E. 1999, A\&A, 343, 558

Kameya, O., Hasegawa, T. I., Hirano, N., Takakubo, K., \& Seki, M. 1989, ApJ, 339, 222

Kameya, O., Hasegawa, T. I., Hirano, N., Tosa, M., Taniguchi, Y., Takakubo, K., \& Seki, M. 1986, PASJ, 38, 793

Kameya, O., Hirano, N., Kawabe, R., \& Campbell, B. 1990a, in Proc. 7th Manchester Astronomical Conference on Molecular Clouds, ed. R. James \& T. Millar (Manchester: Manchester Astron. Soc.), 111

Kameya, O., Morita, K.-I., Kawabe, R., \& Ishiguro, M. 1990b, ApJ, 355, 562

Kameya, O., \& Takakubo, K. 1988, PASJ, 40, 413

Kawabe, R., et al. 1992, PASJ, 44, 435

Königl, A., \& Ruden, S. P. 1993, in Protostars and Planets III, ed. E. H. Levy \& J. I. Lunine (Tuscon: Univ. Arizona Press), 497

Lucas, P. W., \& Roche, P. F. 1998, MNRAS, 299, 699

Matthews, B. C., \& Wilson, C. 2000, ApJ, 531, 868

Mestel, L. 1985, in Protostars and Planets II, ed. D. C. Black \& M. S. Matthews (Tuscon: Univ. Arizona Press), 320
Minchin, N. R, \& Murray, A. G. 1994, A\&A, 286, 579

Nakano, T., \& Nakamura, T. 1978, PASJ, 30, 671

Nakano, T., \& Umebayashi, T. 1986, MNRAS, 218, 663

Sandell, G. 1994, MNRAS, 271, 75

Schleuning, D. A., Vaillancourt, J. E., Hildebrand, R. H., Dowell, C. D., Novak, G., Dotson, J. L., \& Davidson, J. A. 2000, ApJ, 535, 913

Scott, E. H., \& Black, D. C. 1980, ApJ, 239, 166

Scoville, N. Z., Sargent, A. I., Sanders, D. B., Claussen, M. J., Masson, C. R., Lo, K. Y., \& Phillips, T. G. 1986, ApJ, 303, 416

Shu, F. H., Adams, F. C., \& Lizano, S. 1987, ARA\&A, 25, 23

Snell, R. L., Loren, R. B., \& Plambeck, R. L. 1980, ApJ, 239, L17

Tamura, M., Gatley, I., Joyce, R. R., Ueno, M., Suto, H., \& Sekiguchi, M. 1991, ApJ, 378, 611

Tamura, M., Hough, J. H., Greaves, J. S., Morino, J.-I., Chrysostomou, A., Holland, W. S., \& Momose, M. 1999, ApJ, 525, 832

Vallée, J. P., \& Bastien, P. 2000, ApJ, 530, 806

Werner, M. W., Becklin, E. E., Gatley, I., Matthews, K., Neugebauer, G., \& Wynn-Williams, C. G. 1979, MNRAS, 188, 463

Wynn-Williams, C. G., Becklin, E. E., \& Neugebauer, G. 1974a, ApJ, 187, 473

Wynn-Williams, C. G., Werner, M. W., \& Wilson, W. J. 1974b, ApJ, 187, 41 